\title{
Quench of Single-Layer REBCO CORC Cable with Non-Uniform Terminal Contact Resistances
}

\author{
Zixuan Zhu, Yawei Wang, Dong Xing, Xiaoze Pei, Min Zhang, Weijia Yuan
}

\begin{abstract}
ReBCO conductor-on-round-core (CORC) cable has become a promising candidate for high temperature superconducting (HTS) power applications, due to its great mechanical strength, high current carrying capacity, high flexibility and low AC losses. However, ReBCO coated conductors are at risk of quenching, which significantly affects the thermal stability and reliability of the CORC cable. Three-dimensional (3D) numerical study on the quench behavior of the CORC cable remains a challenge, for its complex geometry is difficult to cope with. In this paper, a 3D timedependent multi-physics quench model based on the T-A formulation has been developed. Three modules are coupled in this model; The T-A formulation model, a heat transfer model and an equivalent circuit model. The quench behavior of a single-layer ReBCO CORC cable with non-uniform terminal contact resistances has been studied, when a hotspot is imposed on one of the tapes to induce a local quench. Results show that, the CORC cable has the highest MQE, in another word, is most stable, when the hotspotinduced quench occurs on the tape with the middle value of terminal contact resistance.
\end{abstract}

Index Terms-CORC cable, non-uniform terminal contact resistance, 3D quench modeling, T-A formulation.

\section{INTRODUCTION}

$\mathbf{S}^{\mathrm{E}}$ EVERAL high temperature superconducting (HTS) materials, including $\mathrm{Bi}-2212$, Bi-2223, and (Re)Ba2Ca3O7-x ( $\mathrm{ReBCO}$ ) coated conductors, are widely used in some industrial HTS power applications (e.g., motors/generators, transformers, fault current limiters and transmission cables), due to their high current carrying capacity and high critical magnetic fields [1-3]. The power grid and high field magnets have called for practical HTS cables with high current and low inductance [4-7]. Several ReBCO based cabling concepts are in development, these include the Roebel Assembled coated conductor (RACC) cables [8, 9], Twisted Stacked-Tape cables [10], and Conductor on Round Core (CORC) cables [11-16].

\footnotetext{
Manuscript receipt and acceptance dates will be inserted here. Acknowledgment of support is placed in this paragraph as well.

Zixuan Zhu is with the Department of Electric and Electrical Engineering, University of Strathclyde, Glasgow, U.K. She is also with the Department of Electric and Electrical Engineering, University of Bath, Bath BA2 7AY, U.K. (Corresponding author: Weijia, Yuan.)

Yawei Wang, Min Zhang, Weijia Yuan are with the Department of Electric and Electrical Engineering, University of Strathclyde, Glasgow, UK. (e-mail: weijia.yuan@strath.ac.uk).

Dong Xing and Xiaoze Pei are with the Department of Electric and Electrical Engineering, University of Bath, Bath BA2 7AY, UK.

Color versions of one or more of the figures in this paper are available online at http://ieeexplore.ieee.org.

Digital Object Identifier will be inserted here upon acceptance.
}

The CORC cable shows a great potential in high-field magnets and power devices for its advantages on mechanical strength, high current carrying capacity, mechanic flexibility, and low AC losses [15]. In spite of the progress that has been done in the development of ReBCO coated conductors, the stability and reliability of HTS applications are at risk of quenching. Quench behavior of single ReBCO tapes has been studied a lot [17]. The quench behavior of CORC cable differs from that of a single ReBCO tape, due to the complex structure and current redistribution through terminal contact resistances. Experiments have been carried out to test the stability and normal zone propagation of CORC cable [18, 19]. In practical applications, ReBCO tapes are soldered at terminal leads, the non-uniform contact resistances may has a significant influence on the thermal stability of CORC cables during quench [20], which has not been elucidated in details yet.

This paper presents a study on the quench behavior of a single-layer ReBCO CORC cable with non-uniform terminal contact resistances. To conduct this research, a 3D multiphysics model is built using the finite element method (FEM) software, COMSOL Multiphysics. Three modules are coupled in this model: The T-A formulation module, an equivalent circuit module, and a heat transfer module. A hotspot is induced on each one of the three ReBCO tapes in the cable, causing heat disturbance during its operating process. Temperature rise in each tape, tape voltage variation and the current redistribution among tapes are analyzed. Minimum quench energy (MQE) of the CORC cable with non-uniform terminal contact resistances is calculated.

\section{CABle Model AND NuMERICAL METHOD}

\section{A. Cable model studied}

Fabrication of the HTS CORC cable is to wind ReBCO coated conductors in a helical fashion around a round copper former with relatively small radius. Here, radius of the singlelayer CORC cable is $2.6 \mathrm{~mm}$. Winding angle of the ReBCO tapes is $40^{\circ}$. The ReBCO tape used in this study has a width of $4 \mathrm{~mm}$ and a thickness of $1 \mathrm{~mm}$. More parameters of the ReBCO tape (thermal conductivity, heat capacity, I-V curve of the ReBCO film, etc.) can be seen from [21]. The operating temperature is $70 \mathrm{~K}$, and the critical current of the tape under this temperature is around 148 A. To simplify the simulation, only the superconducting layer is simulated, and ReBCO tapes are assumed to be isolated from each other. During the simula- 
tion process, a heat pulse is applied on one of the three tapes to cause a local hot spot. The 3D geometry of the CORC cable and the area where the heat pulse is imposed are shown in Fig. 1. Detailed specification of this cable is listed in Table I.

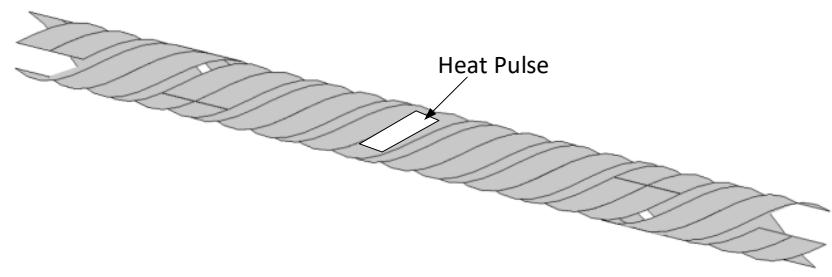

Fig. 1. Geometry of the single-layer CORC cable with three ReBCO tapes and the heat pulse zone.

\section{B. Numerical method}

To build a 3D quench model, three modules are coupled using COMSOL Multiphysics; the T-A formulation model, a heat transfer model on thin shell, and an equivalent circuit model. The numerical algorithm can be seen in Fig. 2 .

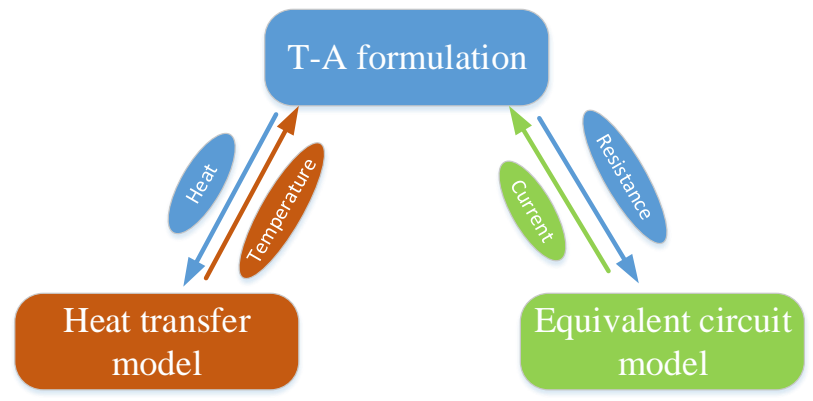

Fig. 2. Numerical algorithm of the 3D quench model

\section{The T-A formulation}

Principle of the T- and A-formulation model is the assumption that the superconducting tape can be approximated as an infinitely thin sheet, which means the thickness of the tape is neglected, due to its high aspect ratio. Thus, meshing number of the model is reduced significantly, which can effectively shorten the computational time. In the T-A formulation, the current vector potential $\mathbf{T}$, which only applies to the superconducting region, is used to calculate the current distribution of the ReBCO tapes. The magnetic vector potential $\mathbf{A}$, on the other hand, is applied to the whole domain to solve the distribution of magnetic field. The obtained magnetic field is then fed back to the T-formulation model to achieve the electromagnetic coupling. Governing equation of the T-A formulation can be expressed as[22-25],

$$
\left\{\begin{array}{l}
\nabla \times \mathbf{E}(\nabla \times \mathbf{T})=\frac{\partial \mathbf{B}}{\partial t} \\
\nabla \times \nabla \times \mathbf{A}=\mu_{0} \mathbf{J}
\end{array}\right.
$$

The E-J relationship of ReBCO tape used here is as follow:
TABLE I

SPECIFICATION OF THE CORC CABLE MODEL

\begin{tabular}{ll}
\hline \hline Parameter & Quantity \\
\hline \hline & Cable \\
\hline Radius & $2.6 \mathrm{~mm}$ \\
Winding angle & $40^{\circ}$ \\
Pitch & $19 \mathrm{~mm}$ \\
Operating current & $360 \mathrm{~A}$ \\
Operating temperature & $70 \mathrm{~K}$ \\
Tapes per layer & 3 \\
\hline & \\
& ReBCO tape \\
Width/Thickness & $4 \mathrm{~mm} / 0.1 \mathrm{~mm}$ \\
Critical current & $148 \mathrm{~A} \mathrm{@} 70 \mathrm{~K}$ \\
Operating current & $120 \mathrm{~A}$ \\
Substrate (Hastelloy) & $50 \mu \mathrm{m}$ \\
Stabilizer (Copper) & $20 \mu \mathrm{m}$
\end{tabular}

$$
\mathbf{E}(\mathbf{J})=E_{0}\left(\frac{|\mathbf{J}|}{J_{c}\left(\mathbf{B}, T_{h}\right)}\right)^{n} \frac{\mathbf{J}}{J_{c}\left(\mathbf{B}, T_{h}\right)}
$$

where the critical electric field $E_{0}=10^{-4} \mathrm{~V} / \mathrm{m}$, and the $E-J$ power law index $n=31$ in this manuscript.

The dependence of the critical current on the temperature and external magnetic field can be expressed as [21, 26-28]:

$$
\begin{aligned}
& J_{c T}\left(T_{h}\right)= \begin{cases}\left(\frac{T_{c}-T_{h}}{T_{c}-T_{o}}\right)^{\beta} & \text { if } T_{h}<T_{c} \\
0 & \text { if } T_{h} \geq T_{c}\end{cases} \\
& J_{c \mathrm{~B}}(B, \theta)=\frac{1}{\left[1+\sqrt{\left(k B_{p a r}\right)^{2}+B_{p e r}^{2}} / B_{c}\right]^{b}}
\end{aligned}
$$

In (3), $\beta$ is the power index in the $J-T$ relation. Operating temperature $T_{o}=70 \mathrm{~K}$, and $T_{c}=92 \mathrm{~K}$ is the critical temperature. In (4), $B_{\text {par }}$ and $B_{\text {per }}$ represent the magnetic fields parallel and perpendicular to the tape surface respectively. $\mathrm{K}=0.0605$, $\mathrm{b}=0.7580$, and $\mathrm{B}_{\mathrm{c}}$ is the critical magnetic field.

\section{The heat transfer model}

A heat transfer in thin shells model is coupled to the T-A formulation model to calculate the temperature of ReBCO tapes. Typical governing equation of the heat transfer model is expressed as follow [21, 26],

$$
\rho C_{p} \frac{\partial T_{s}}{\partial t}+\nabla \cdot\left(-k \nabla T_{s}\right)=Q_{s}+Q_{p}
$$

Here, $\rho$ represents the density of the ReBCO conductors, $T_{s}$ is the temperature of the superconductor. $C_{p}$ and $k$ are the heat capacity and the thermal conductivity respectively, which are both temperature dependent [21]. $Q_{s}$ represents the heat power caused by the transport current flowing in the CORC cable, 
while $Q_{p}$ is an external heat pulse power, which is generated by imposed heater.

The thin sheet approximation is also applied to this model. Although the thermal conductivity of the hastelloy layer in the $\mathrm{ReBCO}$ tape is much lower than that of the copper layer, it is relatively higher than other materials. The heat pulse is relatively long for thermal process. Therefore, it is reasonable to neglect the temperature difference along thickness direction. Thus, a uniform temperature distribution approximation is achieved [26].

\section{E. Geometry of CORC cable \& the equivalent circuit model}

As can be seen from Fig. 3, to measure the current redistribution among ReBCO tapes during the quench process, an equivalent circuit model is built and coupled with other models. Each tape is treated as three components, terminal contact resistance $R_{t}$, total resistance of the tape $\left(R_{\text {tape }}\right)$, and an inductance. Due to the symmetric structure of the CORC cable, the mutual inductances between tapes and the self-inductance of each tape are almost equal. Thus, the current redistribution among tapes is mainly determined by the tape voltage $R_{\text {tape }}$ and the terminal contact resistance $R_{t}$, especially at steady situation.

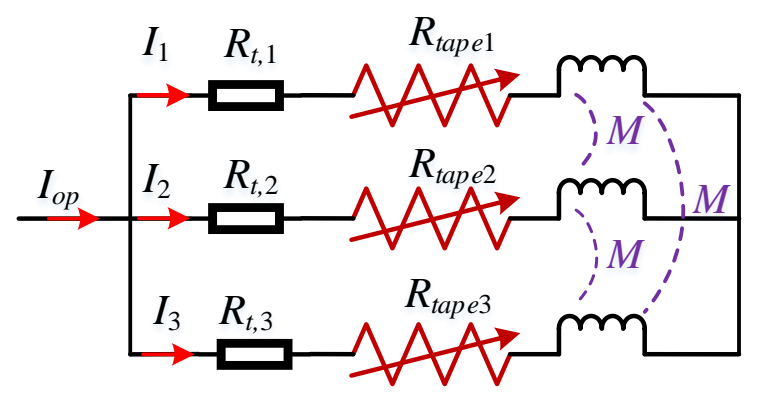

Fig. 3. Equivalent circuit model of the single-layer CORC cable with three ReBCO tapes

\section{RESULTS AND DISCUSSION}

During the simulation process, the single-layer CORC cable carries a total transport current of 360 A. Due to the nonuniform terminal contact resistances, ramped current in each tape is distributed inhomogeneously. Then, a series of heat pulses are imposed on one of the three tapes from $2.0 \mathrm{~s}$, which is called 'the target tape' in this paper, for a period of time to induce a hotspot on that tape. Here, the heat pulse is imposed after two seconds of operation, and duration of the heat pulse is set to be $100 \mathrm{~ms}$. The other two tapes without hotspot are called 'the normal tapes'. Cooling is not considered in this paper. To analyze the quench behavior of a CORC cable with the non-uniform terminal contact resistance $R_{t}$ when a hotspotinduced quench occurs, simulation was performed under a set of non-uniform terminal contact resistances: $R_{t, 1}=40 \mu \Omega, R_{t, 2}$ $=50 \mu \Omega R_{t, 3}=60 \mu \Omega$. Three cases will be studied, in which hotspot is induced on tapes with a terminal contact resistance of $40 \mu \Omega, 50 \mu \Omega$ and $60 \mu \Omega$ respectively.
As shown in Table II, when the heat pulse is imposed on the tape with lowest terminal contact resistance $\left(R_{t}=40 \mu \Omega\right)$, the minimum quench energy (MQE) is $104.4 \mathrm{~mJ}$, while the MQE for the tape with highest terminal contact resistance $\left(R_{t}=60 \mu \Omega\right)$ rises to $122.7 \mathrm{~mJ}$. However, the highest MQE is obtained when the tape with a terminal contact resistance of $50 \mu \Omega$ endures a local quench, which reaches $156.7 \mathrm{~mJ}$. To understand this result, the transport current, tape voltage, and temperature of the CORC cable during the quenching process have been plotted in Fig. 4. Notice that the tape voltage does not include that on terminal contact resistances.

TABLE II

MINIMUM QUENCH ENERGY

\begin{tabular}{cc}
\hline \hline Terminal Contact Resistance $(\mu \Omega)$ & Minimum Quench Energy $(\mathrm{mJ})$ \\
\hline 40 & 104.4 \\
50 & 156.7 \\
60 & 122.7 \\
\hline
\end{tabular}

This table lists the minimum quench energy when the hotspot is induced on tapes with $R_{t}=40 \mu \Omega, 50 \mu \Omega$ and $60 \mu \Omega$ respectively.

Simulation results from Fig. 4 are obtained from the situation when the heat pulse is high enough to cause a local quench on the target tape. Left column presents the current redistribution among tapes, middle column plots the tape voltage of each tape (the voltage of terminal contact resistance is not included), and right column shows the temperature change in each tape. Results show that, after the hotspot is induced, temperature and tape voltage of the target tape both increase rapidly, leading to current decrease in the target tape. Transporting current in the target tape is then forced out to other tapes. This current redistribution is absorbed by normal tapes, causing current rise in these tapes. However, since the tape voltage in target tape is much higher than that of the normal tapes right after the heat pulse is turned off, this voltage rise prevents current from further redistributing to normal tapes. As a result, current in the target tape decreases much slower than before, leading to heat accumulation and higher temperature on the target tape, thus, eventually inducing a quench.

In Fig. $4\left(\mathrm{a}_{1}\right)$, when $R_{t}$ of the target tape is the lowest among all tapes, which, in this case, is $40 \mu \Omega$, current in the target tape drops from 140 A to $80 \mathrm{~A}$, which means that around $43 \%$ of the current is redistributed to normal tapes. Transport current of the tape with $R_{t}=50 \mu \Omega$ rises above its critical value of $148 \mathrm{~A}$, then decrease slightly, back to a value that is lower than $148 \mathrm{~A}$ after the heat pulse is turned off, which leads to a slight voltage rise in this tape. Voltage of the tape with highest $R_{t}(=60 \mu \Omega)$ remains the same for the first 3 seconds, for current in this tape only rises to $130 \mathrm{~A}$ with the heat pulse imposed. In Fig. 4(a $\left.a_{3}\right)$, temperature of the target tape decreases slightly after the heat pulse is turned off, then remains nearly unchanged for a while, before it sharply increases to over 100 $\mathrm{K}$ after $3 \mathrm{~s}$.

From Fig. 4(c), currents in normal tapes rise above their critical values right after the heat pulse is imposed, leading to considerable voltage rise in these tapes. The redistributed current from the target tape leads to continuous over-currents 
flowing in normal tapes. In Fig. 4( $\left.c_{3}\right)$, the temperature of the target tape shows a slight decrease after the heat pulse is turned off, then there's a sudden rise of temperature at around $2.4 \mathrm{~s}$, and the temperature of target tape reaches $100 \mathrm{~K}$ in $0.2 \mathrm{~s}$.

Compared with Fig. 4(a) and (c), quenching process in Fig. 4(b), which is the case when the heat pulse is imposed on the tape with middle value of $R_{t}(40 \mu \Omega)$, is much smoother and shows no sudden change during the whole quenching process. Around $50 \%$ of transporting current is redistributed from the target tape at the end of the heat pulse.

The MQE of a tape in this CORC cable model are determined by two factors, transport current and current redistribu- tion through terminal contact resistances. Lower terminal contact resistance means easier current redistribution to other tapes, which leads to higher MQE. However, it also leads to higher transport current during the ramping of the cable's total current, and tapes with higher transport current has lower MQE. Together, these two factors lead to the conclusion that the tapes with lowest terminal contact resistance does not have largest MQE. Results also show that, for a single-layer CORC cable with non-uniform terminal contact resistances, the tape with moderate terminal contact resistance has highest MQE and best thermal stability.
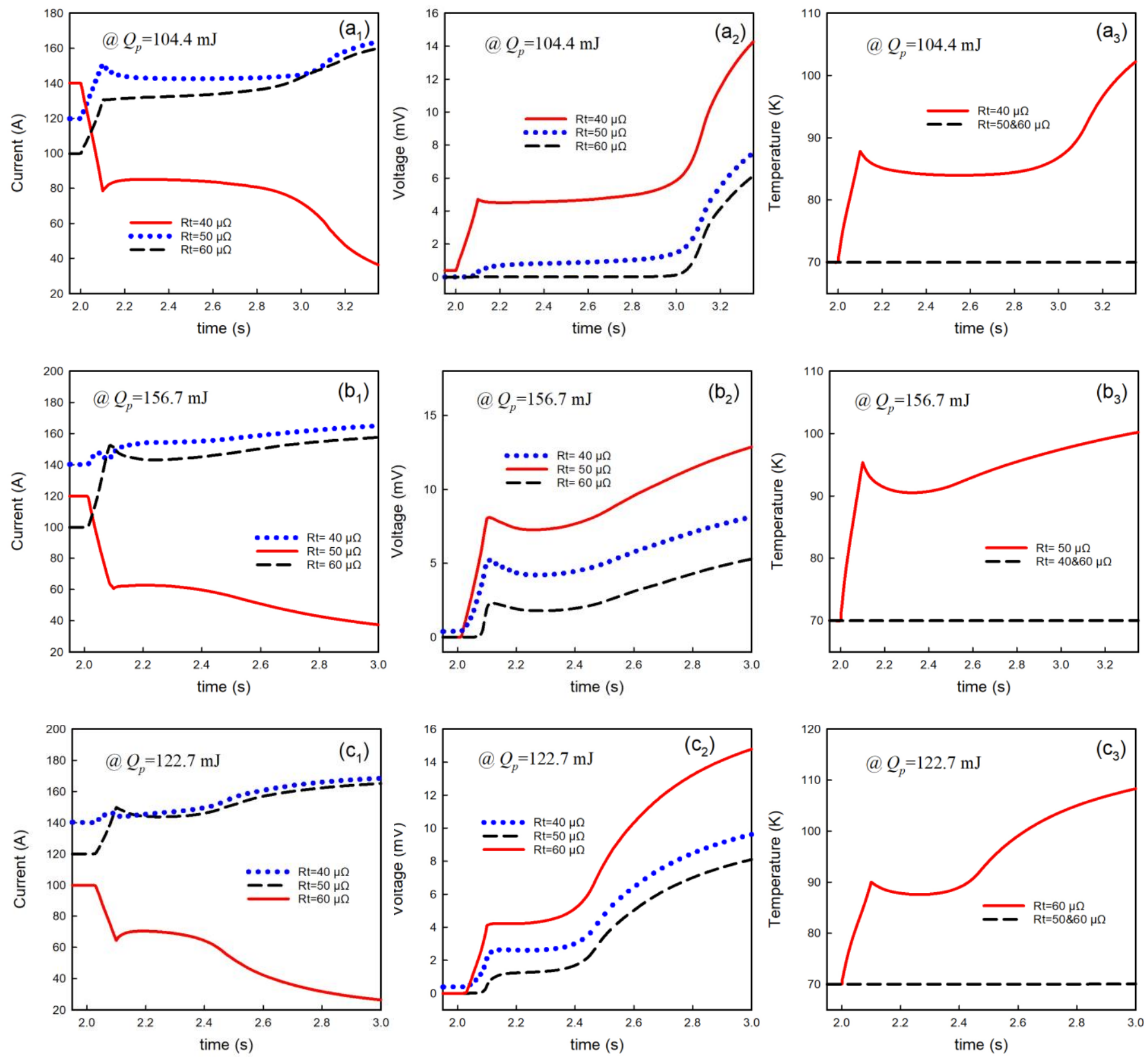

Fig. 4. Current redistribution among tapes, tape voltage, and temperature on each tape during local hotspot-induced quench process, when the heat pulse is imposed on the tape with (a) $R_{t}=40 \mu \Omega$; (b) $R_{t}=50 \mu \Omega$; and (c) $R_{t}=60 \mu \Omega$. 


\section{CONCLUSION}

A 3D multi-physics quench model is developed in this paper to analyze the quench behavior of a single-layer ReBCO CORC cable with non-uniform terminal contact resistances $\left(R_{t}=40 \mu \Omega, 50 \mu \Omega\right.$ and $\left.60 \mu \Omega\right)$. Three modules are coupled in this model: The T-A formulation model, a heat transfer on thin shell model, and an equivalent circuit model. A hotspot is induced on each tape of the cable separately to study their quench behavior.

Results show that, the CORC cable is most stable when a hotspot is induced on the tape with the middle value of terminal contact resistance, in this case, when $R_{t}=50 \mu \Omega$. The highest MQE is also obtained from this case. This is because that, high terminal contact resistance means hard current redistribution and sharp increase of tape voltage, which decreases the thermal stability. Although low terminal contact resistance leads to an easy current redistribution among tapes, it also leads to higher transport current, which lowers the MQE. Thus, conclusion can be made that, for a single-layer ReBCO CORC cable with non-uniform terminal contact resistances, tape with middle value of $R_{t}$ has the largest MQE.

\section{REFERENCES}

[1] M. Zhang, F. Eastham, and W. J. Yuan, "Design and Modeling of 2G HTS Armature Winding for Electric Aircraft Propulsion Applications," Ieee Transactions on Applied Superconductivity, vol. 26, no. 3, Apr 2016, Art. no. 5205705.

[2] R. H. Qu, Y. Z. Liu, and J. Wang, "Review of Superconducting Generator Topologies for Direct-Drive Wind Turbines," Ieee Transactions on Applied Superconductivity, vol. 23, no. 3, Jun 2013, Art. no. 5201108 .

[3] Y. Wang et al., "Analysis and Comparison Between No-Insulation and Metallic Insulation REBCO Magnet for the Engineering Design of a 1MW DC Induction Heater," IEEE Transactions on Applied Superconductivity, vol. 27, no. 4, pp. 1-5, 2017.

[4] S. Akita, "Superconducting technologies in electric power application," 2004 International DAPAS Workshop, Apr. 20042004.

[5] C. Jeonwook et al., "Development and testing of $30 \mathrm{~m}$ HTS power transmission cable," IEEE Transactions on Applied Superconductivity, vol. 15, no. 2, pp. 1719-1722, 2005.

[6] W. Xiaorong et al., "A viable dipole magnet concept with REBCO CORC (B) wires and further development needs for high-field magnet applications," Superconductor Science and Technology, vol. 31, no. 4, p. 045007, 2018.

[7] S. X. Zhang, H. Z. Cheng, D. Wang, L. B. Zhang, F. R. Li, and L. Z. Yao, "Distributed generation planning in active distribution network considering demand side management and network reconfiguration," Applied Energy, vol. 228, pp. 1921-1936, Oct 2018.

[8] W. Goldacker et al., "ROEBEL assembled coated conductors (RACC): Preparation, properties and progress," Ieee Transactions on Applied Superconductivity, vol. 17, no. 2, pp. 3398-3401, Jun 2007.

[9] W. Goldacker, F. Grilli, E. Pardo, A. Kario, S. I. Schlachter, and M. Vojenciak, "Roebel cables from REBCO coated conductors: a onecentury-old concept for the superconductivity of the future," Superconductor Science \& Technology, vol. 27, no. 9, Sep 2014, Art. no. 093001.

[10] M. Takayasu, L. Chiesa, L. Bromberg, and J. V. Minervini, "HTS twisted stacked-tape cable conductor," Superconductor Science \& Technology, vol. 25, no. 1, Jan 2012, Art. no. 014011.

[11] D. C. v. d. Laan, "YBa $2 \mathrm{Cu} 3 \mathrm{O} 7-\delta$ coated conductor cabling for low ac-loss and high-field magnet applications," Superconductor Science and Technology, vol. 22, no. 6, p. 065013, 2009.

[12] D. C. v. d. Laan, X. F. Lu, and L. F. Goodrich, "Compact GdBa 2 Cu 3 O $7-\delta$ coated conductor cables for electric power transmission and magnet applications," Superconductor Science and Technology, vol. 24, no. 4 , p. $042001,2011$.

[13] T. Mulder et al., "Design and Manufacturing of a $45 \mathrm{kA}$ at $10 \mathrm{~T}$ REBCO-CORC Cable-in-Conduit Conductor for Large-Scale Magnets," Ieee Transactions on Applied Superconductivity, vol. 26, no. 4, Jun 2016, Art. no. 4803605 .

[14] D. C. van der Laan et al., "Record current density of 344Amm(-2) at 4.2K and $17 \mathrm{~T}$ in CORC (R) accelerator magnet cables," Superconductor Science \& Technology, vol. 29, no. 5, May 2016, Art. no. 055009.

[15] J. D. Weiss, T. Mulder, H. J. ten Kate, and D. C. van der Laan, "Introduction of CORC (R) wires: highly flexible, round hightemperature superconducting wires for magnet and power transmission applications," Superconductor Science \& Technology, vol. 30, no. 1, Jan 2017, Art. no. 014002.

[16] J. Zhenan, A. Naoyuki, and N. Masaaki, "Numerical calculation of AC losses in multi-layer superconducting cables composed of coated conductors," Superconductor Science and Technology, vol. 21, no. 2, p. 025013, 2008

[17] H. Song and J. Schwartz, "Stability and Quench Behavior of YBa2Cu3O7-x Coated Conductor at 4.2 K, Self-Field," Ieee Transactions on Applied Superconductivity, vol. 19, no. 5, pp. 37353743 , Oct 2009

[18] M. Majoros, M. D. Sumption, E. W. Collings, and D. v. d. Laan, "Stability and normal zone propagation in YBCO CORC cables," Superconductor Science and Technology, vol. 29, no. 4, p. 044006, 2016.

[19] T. Mulder, A. Dudarev, M. Mentink, D. v. d. Laan, M. Dhallé, and H. t. Kate, "Performance Test of an 8 kA @ 10-T 4.2-K ReBCO-CORC Cable," IEEE Transactions on Applied Superconductivity, vol. 26, no. 4, pp. 1-5, 2016.

[20] G. P. Willering, D. C. van der Laan, H. W. Weijers, P. D. Noyes, G. E. Miller, and Y. Viouchkov, "Effect of variations in terminal contact resistances on the current distribution in high-temperature superconducting cables," Superconductor Science \& Technology, vol. 28, no. 3, Mar 2015, Art. no. 035001

[21] Y. Wang, C. Wan Kan, and J. Schwartz, "Self-protection mechanisms in no-insulation (RE) Ba $2 \mathrm{Cu} 3 \mathrm{O} \times$ high temperature superconductor pancake coils," Superconductor Science and Technology, vol. 29, no. 4, pp. 045007 (11 pp.)-045007 (11 pp.), April 2016.

[22] F. Liang et al., "A finite element model for simulating second generation high temperature superconducting coils/stacks with large number of turns," Journal of Applied Physics, vol. 122, no. 4, p. 043903, 2017.

[23] H. Zhang, M. Zhang, and W. Yuan, "An efficient 3D finite element method model based on the T-A formulation for superconducting coated conductors," Superconductor Science and Technology, vol. 30, no. 2, p. 024005, 2016.

[24] Z. Zhu, Y. Wang, S. Venuturumilli, J. Sheng, M. Zhang, and W. Yuan, "Influence of Harmonic Current on Magnetization Loss of a Triaxial CORC REBCO Cable for Hybrid Electric Aircraft," IEEE Transactions on Applied Superconductivity, vol. 28, no. 4, pp. 1-5, 2018.

[25] Y. Wang, M. Zhang, F. Grilli, Z. Zhu, and W. Yuan, "Study of the magnetization loss of CORC cables using 3D TA formulation," Superconductor Science and Technology, 2018.

[26] C. Wan Kan, P. J. Masson, C. Luongo, and J. Schwartz, "ThreeDimensional Micrometer-Scale Modeling of Quenching in High-AspectRatio Coated Conductor Tapes-Part I: Model Development and Validation," IEEE Transactions on Applied Superconductivity, vol. 20, no. 6, pp. 2370-80, Dec. 2010.

[27] F. Grilli, F. Sirois, V. M. R. Zermeno, and M. Vojenciak, "SelfConsistent Modeling of the I-c of HTS Devices: How Accurate do Models Really Need to Be?," Ieee Transactions on Applied Superconductivity, vol. 24, no. 6, Dec 2014, Art. no. 8000508.

[28] W. K. Chan, P. J. Masson, C. A. Luongo, and J. Schwartz, "Influence of Inter-Layer Contact Resistances on Quench Propagation in $\mathrm{YBa} 2 \mathrm{Cu} 3 \mathrm{Ox}$ Coated Conductors," (in English), Ieee Transactions on Applied Superconductivity, vol. 19, no. 3, pp. 2490-2495, Jun 2009. 